\title{
Hydroxyapatite Coating on Titanium by Thermal Decomposition
}

\author{
Masaru Akao", Masataka Ohgaki", Noriyuki Nagai' ${ }^{21}$ and Tohru Takagi" \\ ${ }^{1)}$ Institute of Biomaterials and Bioengineering, Tokyo Medical and Dental University, Tokyo, Japan \\ ${ }^{2)}$ Graduate School of Medicine, Dentistry and Pharmaceutical Science, Okayama University, Okayama, Japan \\ 3) Medical Information Bank Corporation, Tokyo, Japan
}

\begin{abstract}
Hydroxyapatite (HA) coatings on Ti implants are made by the physical methods, mainly plasma spraying. We have developed a new chemical coating technique, thermal decomposition method. This method consisted of applying $\mathrm{HA}$ or perovskite $\left(\mathrm{CaTiO}_{3}\right)$ coating solution containing organometals, phosphate ester (only in HA solution), and organic solvents onto the surface of $\mathrm{Ti}$ and then sintered it at $650^{\circ} \mathrm{C}$. The process was repeated several times. The thin and homogeneous layer included an initial $\mathrm{CaTiO}_{3}$ layer with a thickness of 0.6 $\mu \mathrm{m}$ followed by an HA layer $(2.4 \mu \mathrm{m})$ on top. The underlying $\mathrm{CaTiO}_{3}$ layer reduced oxidation of the Ti substrate and enhanced stability of the coating.
\end{abstract}

Keywords: hydroxyapatite coating, thermal decomposition method, perovskite.

\section{Introduction}

Hydroxyapatite (HA) has been widely used in medicine because of its favorable biocompatibility. However, sintered HA has disadvantages such as shaping and low mechanical properties. Many studies have been conducted using HA as a coating on metal and one of the best materials discovered has been the combination of HA and titanium (Ti). The biocompatibility and mechanical properties of HA are excellent when coated on Ti. There are a variety of techniques for HA coating and one of the most common methods is plasma spraying ${ }^{1-2)}$. However, it has numerous drawbacks: 1) application requires an expensive large system; 2) it is difficult to apply on a convoluted surface; 3) HA decomposes into b-TCP and the other second phases under the high temperatures during spraying; 4) the interface between HA and $\mathrm{Ti}$ is fragile; and 5) a thin stable layer (less than $20 \mathrm{~mm}$ ) cannot be obtained. We have developed a new chemical coating technique, called thermal decomposition method ${ }^{3-5}$. This method consists of applying a solution containing organometal, phosphate ester, and organic solvent onto the surface of the Ti and then sintering it at $650^{\circ} \mathrm{C}$. The thin and homogeneous coating with a thickness of 4 $\mathrm{mm}$ is demonstrated ${ }^{5}$. This paper describes a methodology to form a thin and homogeneous layer which includes an initial perovskite $\left(\mathrm{CaTiO}_{3}\right)$ layer followed by an HA layer on top.

\section{Materials and Methods \\ $\mathrm{CaTiO}_{3}$ and HA Coating Solution}

High purity $\mathrm{CaCO}_{3}$ was heated at $1050^{\circ} \mathrm{C}$ for $2 \mathrm{~h}$ to yield $\mathrm{CaO}$. A solution of 2-ethylhexanoic acid was heated at $100 \sim 120^{\circ} \mathrm{C}$, and the $\mathrm{CaO}$ powder was added slowly into the solution. The solution was stirred at this temperatures until all the $\mathrm{CaO}$ was dissolved. After cooling, the resulting calcium 2-ethylhexanoate was dissolved in 1-butanol. Ti tetra-isopropoxide was added to the solution and stirred to form a transparent solution. Thus the $\mathrm{CaTiO}_{3}$ coating solution was made up.

The HA coating solution was prepared with a method similar to

Correspondence to Masaru Akao, Institute of Biomaterials and Bioengineering, Tokyo Medical and Dental University, 2-3-10 Surugadai, Kanda, Chiyoda-ku, Tokyo 101-0062, Japan. Tel: +81-3-5280-8018; Fax: +81-3-5280-8005. E-mail: akao-bcr@tmd.ac.jp that used for $\mathrm{CaTiO}_{3}$. Bis(2-ethylhexyl) hydrogen phosphate and distilled water were added to the above noted calcium 2ethylhexanoate solution in 1-butanol, and then the HA coating solution was made up.

\section{Coating Techniques on Ti plates}

Grade 2 Ti plate $35 \times 35 \times 0.7 \mathrm{~mm}^{3}$ was etched in a boiling $1 \mathrm{~N}$ $\mathrm{HCl}$ solution for $15 \mathrm{~min}$ and washed in distilled water three times. The Ti plate was dipped into the $\mathrm{CaTiO}_{3}$ coating solution for 5 min. The coated plate was centrifuged at $1500 \mathrm{rpm}$ (400 g) for 1 min. Then it was left at room temperature for $10 \mathrm{~min}$, dried at $110^{\circ} \mathrm{C}$ for $20 \mathrm{~min}$, and sintered at $650^{\circ} \mathrm{C}$ for $10 \mathrm{~min}$. The above cycle was repeated three times. The Ti plate was then dipped into the HA coating solution and treated the same way as the $\mathrm{CaTiO}_{3}$. This process was repeated for ten times.

\section{Results and Discussion}

X-ray diffraction (XRD) pattern of the $\mathrm{CaTiO}_{3}$ coated Ti plate (Fig. 1a) indicated that the surface layer contained well-crystallized $\mathrm{CaTiO}_{3}$ and rutile $\left(\mathrm{TiO}_{2}\right)$ due to oxidation of the Ti substrate which showed intense peaks from a-Ti. The relative intensities from rutile were $1 / 10$ compared to those from non-coated Ti plate heated at $650^{\circ} \mathrm{C}$ for $30 \mathrm{~min}$. Thus the $\mathrm{CaTiO}_{3}$ coating reduced oxidation of the Ti substrate.

XRD pattern of the $\mathrm{HA} / \mathrm{CaTiO}_{3}$ coating on the Ti plate (Fig. 1b) exhibited well-crystallized $\mathrm{HA}$ and $\mathrm{CaTiO}_{3}$ and the double layer consisted of $\mathrm{HA}$ and $\mathrm{CaTiO}_{3}$ was confirmed. Weak diffraction peaks from $\mathrm{TiO}_{2}$ due to oxidation of the $\mathrm{Ti}$ and intense peaks from a-Ti were also present in the pattern. No b-TCP was present in this diffraction pattern. In the previous studies ${ }^{4-5)}$ their HA layer included 30\% b-TCP.

The coating thickness was calculated from the weight increase and surface area of the Ti plate before and after coating. The theoretical densities of $\mathrm{HA}$ and $\mathrm{CaTiO}_{3}$ were assumed to be 3.16 and $4.04 \mathrm{~g} / \mathrm{cm}^{3}$, respectively. Thus, thicknesses of the HA and the $\mathrm{CaTiO}_{3}$ layers were calculated to be 2.4 and $0.6 \mathrm{~mm}$, respectively.

Scanning electron microscopy (SEM) showed that after coating of the $\mathrm{CaTiO}_{3}$ and $\mathrm{HA}$, a uniform film was formed on the Ti surface and morphology of the etched Ti surface was well preserved (Fig. 
International symposium of Maxillofacial \& Oral Regenerative Biology in Okayama 2005 2). A cross-sectional view of the coated sample showed that thickness of the coating film was 3 6 mm which was thicker than the calculated value. The HA/Ti interface had intimate contact, but distinction between $\mathrm{CaTiO}_{3}$ and $\mathrm{HA}$ was not clear.

Fig. 3 shows an example of the $\mathrm{HA} / \mathrm{CaTiO}_{3}$ coating applied to commercial dental Ti implants. The double layer which consisted of $\mathrm{HA}$ and $\mathrm{CaTiO}_{3}$ could ensure enhanced stability and compatibility.

\section{References}

1) Radin SR, Ducheyne P. J Mater Sci-Mater M 3:33-42,1992

2) Ukegawa Y. J Jpn Orthop Assoc 66:688-702,1992

3) Hasegawa S. Bull Tokyo Med Dent Univ 43:25-44,1995

4) Zhou P, Hosonuma M, Kobayashi T, Mitsui H, Akao M, Aoki H. Surface Eng 13:320-322,1997.

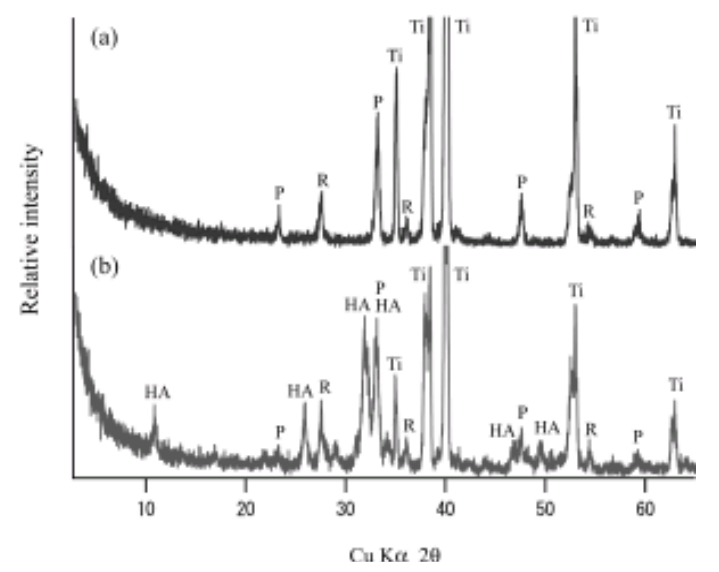

Fig. $1 \mathrm{X}$-ray diffraction patterns of (a) $\mathrm{CaTiO}_{3}$ and (b) $\mathrm{HA} / \mathrm{CaTiO}_{3}$ coating on the Ti plates (Ti: a-Ti; HA: hydroxy-apatite; P: perovskite; R: rutile).

Fig. 3 Example of the $\mathrm{HA} / \mathrm{CaTiO}_{3}$ coating applied to commercial dental Ti implants. 